\title{
Caffeine Maintains Arousal Level and Prevents Change of Electroencephalogram Spectral Powers with Time at Rest
}

\author{
Ji Young Jung ${ }^{1}$, Ho-Won Lee ${ }^{2}$, Il-Sung Jang ${ }^{3}$, Kyoungho Suk ${ }^{1}$, Maan-Gee Lee ${ }^{1}$ \\ ${ }^{1}$ Departments of Pharmacology, ${ }^{2}$ Neurology, School of Medicine and Brain Science and Engineering Institute, \\ Kyungpook National University, Daegu, \\ ${ }^{3}$ Department of Pharmacology, School of Dentistry and Brain Science and Engineering Institute, Kyungpook National University, Daegu, Korea
}

Received December 24, 2013

Revised April 15, 2014

Accepted April 15, 2014

Address for correspondence Maan-Gee Lee, MD

Lab for Control of Brain Activity, Department of Pharmacology,

School of Medicine and

Brain Science and

Engineering Institute,

Kyungpook National University,

Room 516, Biomedical Science

Building, 515-3 Hakjeong-dong,

Buk-gu, Daegu 702-911, Korea

Tel: +82-53-950-4231

E-mail: mglee@knu.ac.kr
Objectives: Although many studies have used quantitative electroencephalography (qEEG) analysis, some inconsistencies remain regarding the effects of caffeine. Among variables producing inconsistencies, the effect of selecting analyzing segment on caffeine study was examined by comparing the first and second halves of a recording session. Methods: Eleven subjects underwent two EEG recording sessions for decaffeinated (control group) and caffeinated coffee (caffeine group) with at least a $48 \mathrm{hr}$ interval. The log absolute powers $(\mathrm{BA})$ and $\log$ relative powers ratio $(\mathrm{BRr})$ of 8 frequency bands were compared between groups and between the first 5-min ( 1 1) and second 5-min (c2) recording segments. Results: As BA's were compared between groups, Delta2 and Theta1 were lower at both segments in the caffeine group and these differences were greater at c2. Increased differences were mainly due to temporal change in the control group. As BRr's were compared, Delta2 was lower at both segments and the difference was greater at $\mathrm{c} 2$ in the caffeine group. Theta1 was lower at c2, Beta BRr was higher at c1, while Alpha2 BRr was higher at $c 2$ in the caffeine group. The dissimilarity indices between groups increased about two-fold with. The dissimilarity indices between segments were about three-times larger in the control group than in the caffeine group. Conclusions: The results indicate that selection of analyzing segment is one important factor for quantitative EEG, and suggest that caffeine prevent decline of arousal, and that the simple 'dissimilarity index' can well represent the effect of caffeine on EEG spectral powers. J Korean Sleep Res Soc 2014;11(1):5-10

Key Words: Band absolute power, Band relative power, Topographic map, Caffeine, Dissimilarity index.

\section{Introduction}

Caffeine is one of the most commonly used central stimulants. It is frequently used for its mood and performance enhancing effects. The daily caffeine intake of Korean university students and North American adults are approximately 120 $\mathrm{mg}$ and $200 \mathrm{mg}$, respectively. ${ }^{1,2}$ Caffeine increases vigilance and diminishes sleepiness, and administration of these doses stimulates attention and restores performance degraded by factors such as fatigue and boredom. ${ }^{3}$

Central stimulating effects of caffeine have been evaluated by quantitative electroencephalography (qEEG) analysis. Although many studies have used qEEG analysis, inconsistencies still remain regarding the effects of caffeine on electroencephalography (EEG) spectral powers. ${ }^{4}$ These inconsistencies

This is an Open Access article distributed under the terms of the Creative Commons Attribution Non-Commercial License (http://creativecommons.org/licenses/by-nc/3.0) which permits unrestricted non-commercial use, distribution, and reproduction in any medium, provided the original work is properly cited. could be attributed to experimental differences between studies. Many studies have been conducted to clarify the effects of various parameters, such as, dose-dependency, ${ }^{5-7}$ eyes open or closed, ${ }^{6,8,9}$ and vigilance control. ${ }^{9}$ However, recording duration for qEEG analysis has not been well considered. According to multiple sleep latency test (MSLT) studies, sleep latency in normal adults during the day is around $15 \mathrm{~min} .{ }^{10}$ Considering that the duration of the EEG recordings are generally 10-30 min, sleep can occur during recording and thus EEG rhythms can change. Accordingly, the selection of a part from a recording for qEEG analysis can affect the outcome of studies on the effects of caffeine on EEG spectral powers. In contrast to subjective questionnaires or neuropsychological tests, qEEG analysis has the advantage of being able to provide objective descriptions of temporal changes.

Therefore, we examined the effect of caffeine effect on temporal change by qEEG by dividing the recording period into two segments and by comparing the qEEG parameters of 
segments. Also, natural vigilance changes were not disturbed by artificial stimulus to a subject.

\section{Methods}

\section{Subjects}

Eleven healthy young adults $\{5$ females and 6 males; age $31 \pm 9$ years [mean \pm standard deviation $(\mathrm{SD})]\}$ participated in this study. The study was conducted in accordance with the Declaration of Helsinki. Written informed consent was obtained from all subjects, and prior approval was obtained from the Institutional Review Board of Kyungpook National University.

\section{Protocol}

Subjects went to bed at their usual time, and they were asked to refrain from caffeine- or alcohol-containing drinks for 24 hours before recording sessions. Each subject underwent two recording sessions: one after drinking decaffeinated (the control group, Maxim Decaf Coffee, DongSuh Inc., Seoul, Korea) and one after caffeinated coffee [the caffeine group, Maxim Coffee, DongSuh Inc., Seoul, Korea; $3 \mathrm{mg}$ caffeine in $1 \mathrm{~mL}$ water per kg body weight; body weight $60.3 \pm 11.3 \mathrm{~kg}$; and caffeine dose $181 \pm 34 \mathrm{mg}(\operatorname{mean} \pm \mathrm{SD})]$. The two sessions were conducted under randomized, double-blind crossover conditions. The interval between the two sessions was at least 48 hours. EEG recording sessions were started $30 \mathrm{~min}$ after drinking and consisted of 2-min eyes closed, 2-min eyes open, 10min eyes closed, and 2-min eyes open periods in sequence. Subjects sat upright in a comfortable chair and followed the instructions of eyes open/closed during the recording session.

\section{Quantitative EEG}

Details of data acquisition and analysis have been described in previous report. ${ }^{11}$ EEG was recorded bipolarly using 21 gold disk electrodes according to the International 10-20 system using $\mathrm{C} 3$ and $\mathrm{C} 4$ as physical references. Signals were amplified and sampled at $500 \mathrm{~Hz}$ (JE-921A and EEG-1200K, Nihon-Kohden, Tokyo, Japan), and the signals of 19 channels were derived by averaged ears reference. Analysis was processed by Hanning window-enveloped and fast Fourier transformation of every 2-sec epoch with 1-sec overlap. Power spectra were calculated with a $0.5 \mathrm{~Hz}$ frequency resolution. Each spectrum was divided into powers at 8 frequency bands: Delta1, 0.5-2.0 Hz; Delta2, 2.5-4.0 Hz; Theta1, 4.5-6.0 Hz; Theta2, 6.5-8.0 Hz; Alpha1, 8.5-11.0 Hz; Alpha2, 11.5-14.0 Hz; Beta, 14.5-25.0 Hz; and Gamma, 25.5-50.0 Hz. Total power was calculated from the entire range. The log absolute powers (BA) and log relative power ratios (BRr) of the 8 frequency bands were calculated from the first ( $\mathrm{cl}$ ) and second (c2) 5-min segments of the 10-min eyes-closed period. BRr was calculated using:

$$
\mathrm{BRr}=\log _{10}\left(\frac{\mathrm{R}}{1-\mathrm{R}}\right)
$$

Where $\mathrm{R}$ is a ratio of the power of each frequency band to total power.

\section{Topographic map}

Maps were based on BA or BRr values of the signals of 19 electrodes. Values were color-coded (ranging from dark blue, through shades of blue, cyan, green, yellow, red to dark red) and plotted at corresponding position on a planar projection of the scalp model. Values between electrodes were interpolated over $50 \times 50$ locations. Each BA map was scaled separately to optimize contrast by using an adequate color range. The difference between maxima and minima in each map was 3 . Each BRr map was scaled using a fixed range from -2 to 0 .

\section{Statistics and comparison}

Differences between band powers were tested using the paired t-test or repeated measures analysis of variance. Differences between topographic maps were expressed as dissimilarity indices $\left(D_{\mathrm{i}}\right)$ between two maps as calculated using:

$$
D_{i}=\sum_{j=1}^{n} \frac{\left(\left|A_{i j}-B_{i j}\right|\right)}{n}
$$

Where, $i$ is the $i$-th band, $j$ is the $j$-th electrode, $A_{i j}$ is a BA or $B R r$ at the $j$-th electrode of the $i$-th band in one group, $B_{i j}$ is a $\mathrm{BA}$ or $\mathrm{BRr}$ at the $\mathrm{j}$-th electrode of the $\mathrm{i}$-th band in another group, and $\mathrm{n}$ is the number of electrodes.

\section{Analysis software}

Quantitative EEG analysis, construction of topographic maps and statistical comparison were done by custom-made software written in Matlab (v2007b, Mathworks, Natick, MA, USA).

\section{Results}

Topographic maps of BA are displayed in Fig. 1. In the caffeine group than in the control group during the first segment (c1), Delta2 BA was significantly lower in the bilateral parietal region (P3, P4) and Theta1 BA in the left parietal region (P3) (Fig. 1 left column). During the second segment (c2), Delta2 BA was significantly lower at the wider area including the midline $(\mathrm{Fz}, \mathrm{Cz}, \mathrm{Pz})$, bilateral central $(\mathrm{C} 3, \mathrm{C} 4)$ and temporal (T3, T4, T6), and bilateral occipital $(\mathrm{O} 1, \mathrm{O} 2)$ 

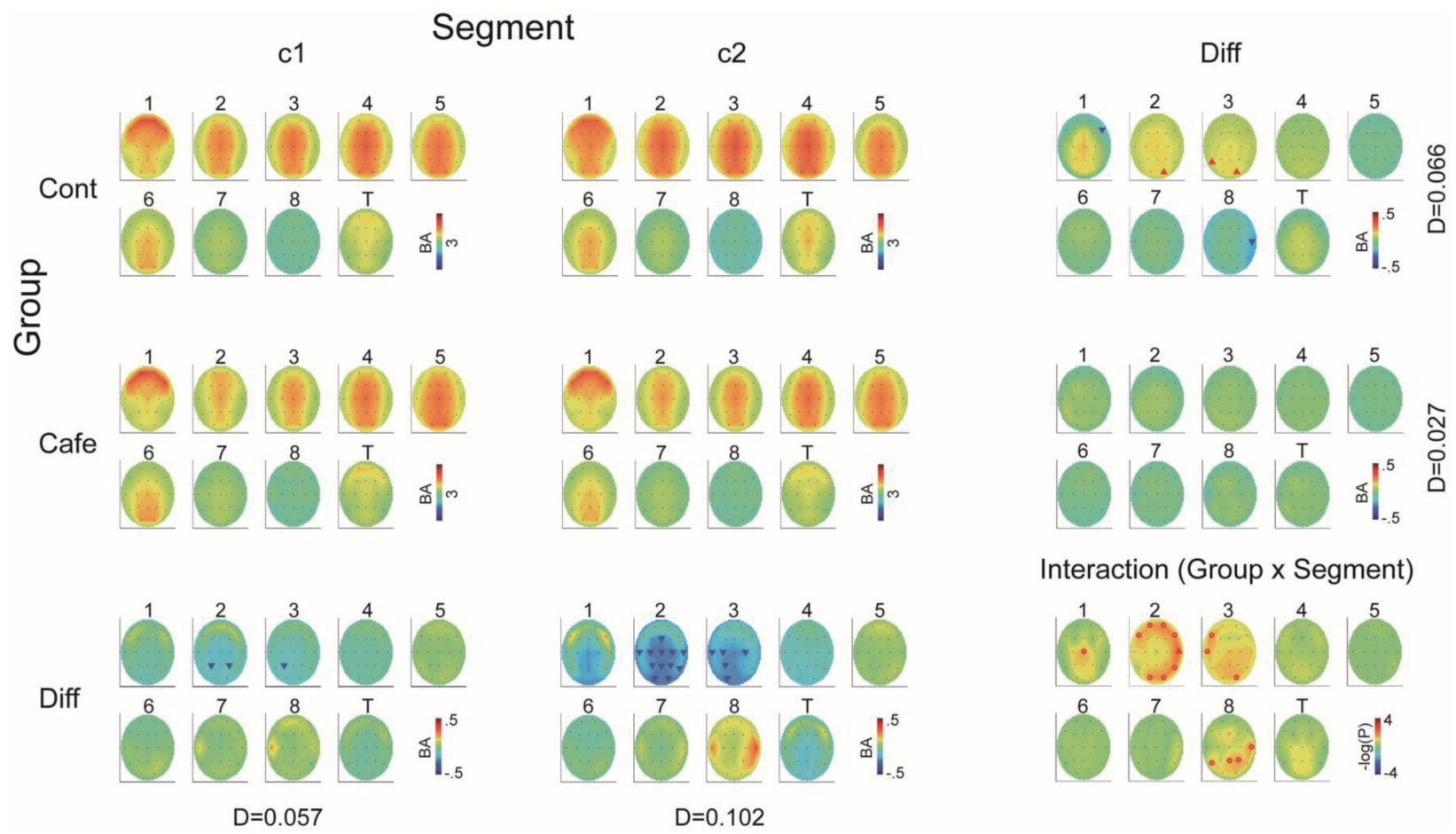

Interaction (Group x Segment)

Figure 1. Topographic maps of log absolute powers at the frequency bands: 1, Delta1 (0.5-2.0 Hz); 2, Delta2 (2.5-4.0 Hz); 3 , Theta1 (4.5-6.0 Hz); 4, Theta2 (6.5-8.0 Hz); 5, Alpha1 (8.5-11.0 Hz); 6, Alpha2 (11.5-14.0 Hz); 7, Beta (14.5-25.0 Hz); and 8, Gamma (25.5-50.0 $\mathrm{Hz}$ ). From top to bottom, maps of the control group (Cont), the caffeine group (Café), and a difference map (Diff). From left to right, maps of the first (c1) and second (c2) 5-min segments, and a difference map (Diff). Triangles and circles indicate $p<0.01$ and $p<0.05$, respectively. Red and blue symbols indicate larger and smaller than the control group or C1, respectively. Small dots indicate the locations of electrodes. Dissimilarity indices (D) between group maps and between segments are shown on the bottom and right, respectively.

regions in addition to the bilateral parietal (P3, P4) region (Fig. 1 middle column).

In the control group across time (c2 compared to $\mathrm{c} 1$ ), Delta2 BA was increased at $\mathrm{O} 2$ and Theta1 BA at $\mathrm{O} 2$ and T5 (Fig. 1 top row). Delta1 BA was decreased at F8 and Gamma BA at T4 (Fig. 1 top row). In contrast, no significant changes were detected in the caffeine group across time (Fig. 1 middle row). Significant interactions between group $\times$ segment were detected mostly in Delta2, Theta1, and Gamma BA (Fig. 1 right bottom panel). The interactions were due to power changes in the control group, whereas no changes were observed in the caffeine group across segments.

Topographic maps of BRr are displayed in Fig. 2. In the caffeine group than in the control group, Delta2 BRr was significantly lower in the posterior (P3, P4, and $\mathrm{O} 1)$ region and Beta $\mathrm{BRr}$ was higher in the right centro-parietal (C4 and $\mathrm{P} 4)$ region during the first segment (c1) (Fig. 2 left column). During the second segment (c2), Delta2 $\mathrm{BRr}$ was lower in most regions (F3, Fz, T3, C3, Cz, C4, T4, T5, Pz, P4, T6, O1, and $\mathrm{O} 2)$ and Theta1 BRr was lower in the central and temporal regions (T3, C3, Cz, C4, and T5) (Fig. 2 middle column). In contrast to those during $\mathrm{cl}$, Alpha2 $\mathrm{BRr}$ was higher in the parieto-occipital region $(\mathrm{Pz}, \mathrm{P} 4, \mathrm{O} 1$, and $\mathrm{O} 2$ ) during $\mathrm{c} 2$ (Fig. 2 middle column).
In the control group across time, Delta1 $\mathrm{BRr}$ was increased at $\mathrm{Cz}$, Delta2 BRr in the fronto-temporal regions (Fp1, Fp2, F7, F4, F8, T4, and T6), Theta1 BRr in the fronto-temporal regions (Fp1, Fp2, F7, F8, T4, and T5), and Theta2 BRr at F7 (Fig. 2 top row). Alpha1 BRr was decreased in F3, Fz, C3, Cz, T5, P3, Pz, P4, O1, and O2 (Fig. 2 top row). In contrast, Theta2 $\mathrm{BRr}$ was increased in only Fp1 across time in the caffeine group (Fig. 2 middle row). Significant interactions between group $\times$ segment were detected mostly in Delta2 and Gamma $\mathrm{BRr}$ (Fig. 2 right bottom panel).

The dissimilarity indices of the topographic maps between groups increased about two-fold with time (from 0.057 to 0.102 in BA, and from 0.057 to 0.100 in BRr) and those between segments were about three-times larger in the control group (0.066 in BA and 0.067 in $\mathrm{BRr}$ ) than in the caffeine group (0.027 in BA and 0.026 in $\mathrm{BRr}$ ).

\section{Discussion}

The important finding is that the differences of band powers in the caffeine and control groups became larger with time and this was attributed to temporal changes of powers in the control group. Absolute powers increased with time in fast delta and slow theta frequencies during rest without caffeine. 


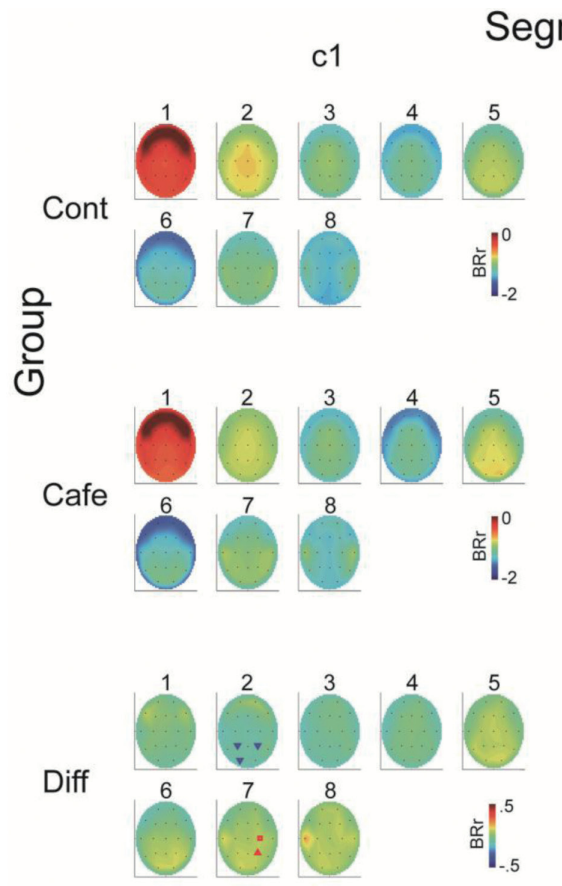

$\mathrm{D}=0.057$
Segment
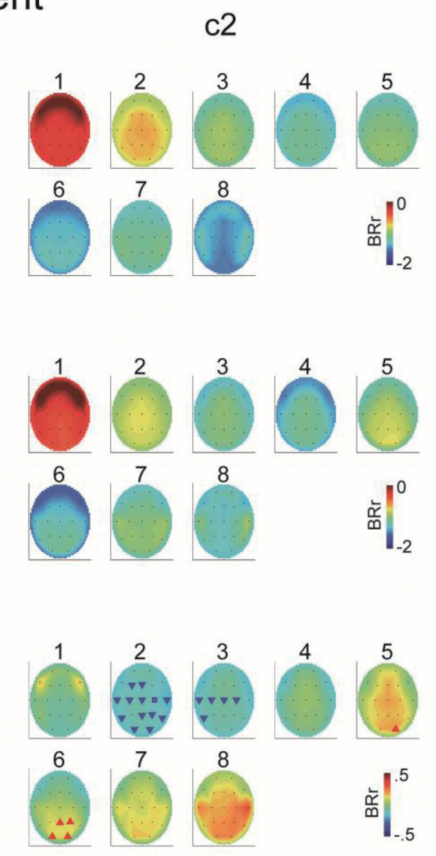

$\mathrm{D}=0.100$
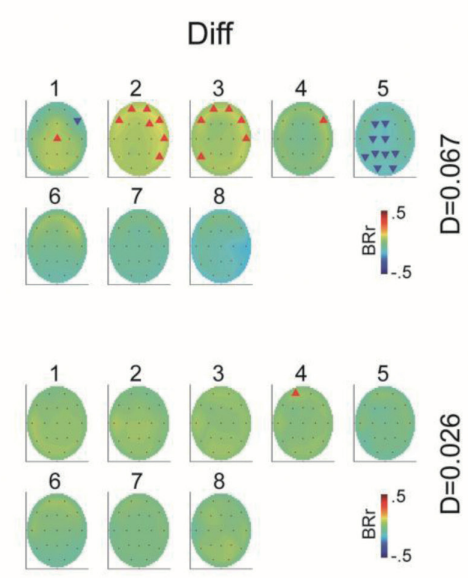

Interaction (Group x Segment)

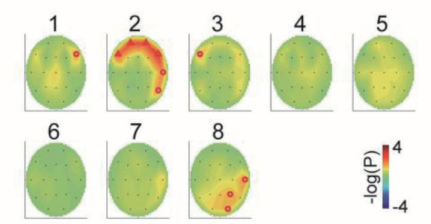

Figure 2. Topographic maps of log relative power ratios at the frequency bands: 1, Delta1 (0.5-2.0 Hz); 2, Delta2 (2.5-4.0 Hz); 3 , Theta1 (4.5-6.0 Hz); 4, Theta2 (6.5-8.0 Hz); 5, Alpha1 (8.5-11.0 Hz); 6, Alpha2 (11.5-14.0 Hz); 7, Beta (14.5-25.0 Hz); and 8, Gamma (25.5-50.0 $\mathrm{Hz}$ ). From top to bottom, maps of the control group (Cont), the caffeine group (Café), and a difference map (Diff). From left to right, maps of the first (c1) and second (c2) 5-min segments, and a difference map (Diff). Triangles and circles indicate $p<0.01$ and $p<0.05$, respectively. Red and blue symbols indicate larger and smaller than the control group or C1, respectively. Small dots indicate the locations of electrodes. Dissimilarity indices (D) between group maps and between segments are shown on the bottom and right, respectively.

Similarly, relative powers increased with time in fast delta and slow theta frequencies and, in addition, were decreased in the slow alpha frequency range. These observations indicate that caffeine maintains arousal level, which changes from the wake state to sleepiness during the rest condition.

Significant differences between the caffeine and control groups were observed only at two parietal electrodes for fast delta power and at one parietal electrode for slow theta power during the first half (segment) of the recording with eyes closed. This difference expanded to 12 electrodes in surrounding regions during the second half (segment) of the recording. Our findings, particularly during the second half, correspond to some studies. Dimpfel et al. reported a decrease in EEG power in the delta and theta ranges after $400 \mathrm{mg}$ caffeine at rest. ${ }^{5}$ Landolt et al. reported theta activity reduction after $200 \mathrm{mg}$ of caffeine. ${ }^{12}$ However, our findings do not agree with those of the following studies. A reduction of alpha power and reduction of beta power have been reported in some studies. ${ }^{5,8,13}$ In the present study, $3 \mathrm{mg} / \mathrm{kg}$ caffeine was used and actually $181 \pm 34 \mathrm{mg}$ caffeine was used. Pritchard et al. using a dose of less than $200 \mathrm{mg}$ ( $2.5 \mathrm{mg} / \mathrm{kg}$ body weight) found little effect of caffeine on EEG. ${ }^{14}$ Other studies by Clubley et al. even observed an increase in delta power, ${ }^{6}$ and Siepmann and Kirch observed a decrease in alpha and slow beta activities. ${ }^{15}$
Reductions of fast delta and slow theta activities were more prominent in the second segment than the first segment of recording. This increase of effect with time might not be due to pharmacokinetics. In this study, caffeine was administered 30 min before the start of recording, and recording was performed from $30 \mathrm{~min}$ to $46 \mathrm{~min}$ after caffeine intake. Caffeine concentration in blood approaches a peak about $30 \mathrm{~min}$ after drinking. ${ }^{16}$ Thus, we assume that EEG recordings were made during the maximal effect of caffeine. However, there is no dose-dependent relationship of caffeine effects on EEG. Therefore, the increased difference between groups with time could not be explained by a gradual increase in caffeine level. In the present study, the power of fast delta and slow theta activity in the control group were significantly different during the first and second halves of recording, while those in the caffeine group were not. Studies of MSLT and sustained attention task undertaken to examine the effects of caffeine on the central nervous system showed caffeine blocks spontaneous decrease of sleep latency during the rest and maintains reaction time and error rate in a longer period..$^{17-19}$ Therefore, significant differences were observed between the caffeine and control groups, especially during the second half, presumably because caffeine blocked spontaneous changes in arousal level during the rest, which manifested as an increase of 
power of fast delta and slow theta ranges in the present study.

James et al. questioned why most studies on the effects of caffeine on EEG did not show caffeine effect but rather showed reversal of caffeine withdrawal of short period. ${ }^{20,21}$ Actually, it was reported that withdrawal of caffeine increase sleepiness and theta power. ${ }^{22,23}$ These results correspond to our findings of increased slow delta and fast theta activities with time after caffeine administration. In our study, abstinence from caffeine for 24 hours before recording was implemented. Considering that the half-life of plasma caffeine is 3 to 10 hours, withdrawal could have occurred in our study. Therefore, we might have observed caffeine withdrawal effect, such as increase of delta and theta activities. Nevertheless, we can conclude that the selection of analysis period from a recording and recording time after caffeine administration can affect results. Our findings suggest that the selection of a recording segment and the choice of recording time after caffeine administration in caffeine studies should be carefully considered.

The relative power of the fast delta range decreased but that of the beta range increased during the first half of recording in the present study. Furthermore, the decrease in fast delta expanded to a wider region and the decrease in slow theta range appeared in the second half of recording. And while increased beta activity disappeared, increased alpha activity appeared during the second half. Because the effect of caffeine on relative EEG power has been little studied, we could not compare the results but only expect a trend similar to absolute power studies. It seems that changes in fast delta and slow theta ranges manifest as changes in relative alpha and beta ranges.

In this study, dissimilarity indices, which represent the difference between topographic maps of absolute powers, were calculated, in addition to the comparison at each electrode in the band power in the caffeine and control groups, and the comparison between the first and second halves of recordings (see the Methods section). The index between the caffeine and control groups increased from 0.057 during the first half of the recording to 0.100 during the second half. The index between the first and second halves was 0.067 in the control group and 0.026 in the caffeine group. The greater dissimilarity during the second half between two groups might have been due to the change in the topographic map of the control group with time rather than being due to caffeine-induced change with time. A significant group $\times$ segment interaction of powers in the fast delta and slow theta ranges was found to result from the change in the control group. Although there was no significant difference of power in the gamma range between the two groups, a significant group $\times$ segment interaction of powers in gamma range was observed. Similar findings were observed in the topographic maps of relative powers.
The results indicate that selection of analyzing segment is one important factor for determining qEEG, and suggest that caffeine can prevent a decline in arousal level and changes in EEG spectral parameters that occur spontaneously during rest. Furthermore, they show that the simple 'dissimilarity index' can well represent the effect of caffeine on EEG spectral powers.

\section{Acknowledgments}

This work was supported by the Basic Science Research Program through the National Research Foundation funded by the Korean Ministry of Education, Science and Technology (2012R1A2A2A02046812).

\section{REFERENCES}

1. Lee BH, Park YS, Kim JS, Yoo JH, Lee JK. Caffeine consumption and its related symptoms in university students. J Korean Acad Fam Med 2007;28:9-16.

2. Wells SJ. Caffeine: implications of recent research for clinical practice. Am J Orthopsychiatry 1984;54:375-389.

3. Weiss B, Laties VG. Enhancement of human performance by caffeine and the amphetamines. Pharmacol Rev 1962;14:1-36.

4. Reeves RR, Struve FA. Topographic brain mapping of caffeine use and caffeine withdrawal. In: Chen FJ. Trends in Brain Mapping Research. New York: Nova Science Publishers, 2006;201-204.

5. Dimpfel W, Schober F, Spüler M. The influence of caffeine on human EEG under resting conditions and during mental loads. Clin Investig 1993;71:197-207.

6. Clubley M, Bye CE, Henson TA, Peck AW, Riddington CJ. Effects of caffeine and cyclizine alone and in combination on human performance, subjective effects and EEG activity. Br J Clin Pharmacol 1979;7:U57U63.

7. Hasenfratz M, Bättig K. Acute dose-effect relationships of caffeine and mental performance, EEG, cardiovascular and subjective parameters. Psychopharmacology (Berl) 1994;114:281-287.

8. Deslandes AC, Veiga H, Cagy M, Piedade R, Pompeu F, Ribeiro P. Effects of caffeine on the electrophysiological, cognitive and motor responses of the central nervous system. Braz J Med Biol Res 2005;38: 1077-1086.

9. Barry RJ, Clarke AR, Johnstone SJ. Caffeine and opening the eyes have additive effects on resting arousal measures. Clin Neurophysiol 2011; 122:2010-2015.

10. Clodoré M, Benoit O, Foret J, Bouard G. The Multiple Sleep Latency Test: individual variability and time of day effect in normal young adults. Sleep 1990;13:385-394.

11. Lee SW. Inter-individual and intra-individual differences of subjects and technicians in repeated EEG measurements. $\mathrm{PhD}$ thesis, Graduate School of Kyungpook National University, 2012.

12. Landolt HP, Rétey JV, Tönz K, et al. Caffeine attenuates waking and sleep electroencephalographic markers of sleep homeostasis in humans. Neuropsychopharmacology 2004;29:1933-1939.

13. Barry RJ, Rushby JA, Wallace MJ, Clarke AR, Johnstone SJ, Zlojutro I. Caffeine effects on resting-state arousal. Clin Neurophysiol 2005;116: 2693-2700.

14. Pritchard WS, Robinson JH, deBethizy JD, Davis RA, Stiles MF. Caffeine and smoking: subjective, performance, and psychophysiological effects. Psychophysiology 1995;32:19-27.

15. Siepmann M, Kirch W. Effects of caffeine on topographic quantitative EEG. Neuropsychobiology 2002;45:161-166.

16. Blanchard J, Sawers SJ. The absolute bioavailability of caffeine in man. Eur J Clin Pharmacol 1983;24:93-98.

17. Bonnet MH, Arand DL. The use of prophylactic naps and caffeine to 
maintain performance during a continuous operation. Ergonomics 1994; 37:1009-1020.

18. Foxe JJ, Morie KP, Laud PJ, Rowson MJ, de Bruin EA, Kelly SP. Assessing the effects of caffeine and theanine on the maintenance of vigilance during a sustained attention task. Neuropharmacology 2012;62:23202327.

19. Zwyghuizen-Doorenbos A, Roehrs TA, Lipschutz L, Timms V, Roth T. Effects of caffeine on alertness. Psychopharmacology (Berl) 1990;100: 36-39.

20. James JE. Caffeine-induced enhancement of cognitive performance: confounding due to reversal of withdrawal effects. Aust J Psychol 2005; 57:197-200.

21. James JE, Keane MA. Caffeine, sleep and wakefulness: implications of new understanding about withdrawal reversal. Hum Psychopharmacol 2007;22:549-558.

22. Keane MA, James JE. Effects of dietary caffeine on EEG, performance and mood when rested and sleep restricted. Hum Psychopharmacol 2008;23:669-680.

23. James JE, Gregg ME. Effects of dietary caffeine on mood when rested and sleep restricted. Hum Psychopharmacol 2004;19:333-341. 